\title{
Wave Function Microscopy of Quasibound Atomic States
}

\author{
S. Cohen, ${ }^{1}$ M. M. Harb, ${ }^{2}$ A. Ollagnier, ${ }^{2}$ F. Robicheaux, ${ }^{3}$ M. J. J. Vrakking, ${ }^{4}$ T. Barillot, ${ }^{2}$ F. Lépine, ${ }^{2, *}$ and C. Bordas ${ }^{2}$ \\ ${ }^{1}$ Physics Department, Atomic and Molecular Physics Laboratory, University of Ioannina, 45110 Ioannina, Greece \\ ${ }^{2}$ Institut Lumière Matière, Université Lyon 1, CNRS, UMR 5306, 10 rue Ada Byron 69622 Villeurbanne Cedex, France \\ ${ }^{3}$ Department of Physics, Auburn University, Auburn, Alabama 36849, USA \\ ${ }^{4}$ Max-Born-Institut, Max Born Straße 2A, D-12489 Berlin, Germany
}

(Received 10 May 2012; revised manuscript received 30 November 2012; published 3 May 2013)

\begin{abstract}
In the 1980s Demkov, Kondratovich, and Ostrovsky and Kondratovich and Ostrovsky proposed an experiment based on the projection of slow electrons emitted by a photoionized atom onto a positionsensitive detector. In the case of resonant excitation, they predicted that the spatial electron distribution on the detector should represent nothing else but a magnified image of the projection of a quasibound electronic state. By exciting lithium atoms in the presence of a static electric field, we present in this Letter the first experimental photoionization wave function microscopy images where signatures of quasibound states are evident. Characteristic resonant features, such as (i) the abrupt change of the number of wave function nodes across a resonance and (ii) the broadening of the outer ring of the image (associated with tunneling ionization), are observed and interpreted via wave packet propagation simulations and recently proposed resonance tunneling mechanisms. The electron spatial distribution measured by our microscope is a direct macroscopic image of the projection of the microscopic squared modulus of the electron wave that is quasibound to the atom and constitutes the first experimental realization of the experiment proposed 30 years ago.
\end{abstract}

DOI: 10.1103/PhysRevLett.110.183001

PACS numbers: $32.80 . \mathrm{Fb}, 32.60 .+\mathrm{i}, 32.80 . \mathrm{Rm}$

The quantum description of atoms and molecules is based on the key concept of the wave function. Traditionally, information on this wave function is inferred by comparing theoretically calculated and experimentally measured observables, such as absorption spectra. With recent experimental progress, an atomic or molecular orbital can be reconstructed and visualized by manipulating electrons with strong laser fields [1] or by photoemission from a molecule deposited on a surface [2]. These experiments pave the way to spectroscopic tools where the interest is focused on the wave function itself. Along these lines, the development of electron imaging techniques has permitted the advent of wave function microscopy, i.e., the direct experimental observation of the projection of the squared modulus of an electronic wave function. This is accomplished by recording the two-dimensional flux of very slow electrons ejected by an ionization process in the presence of a static electric field. In fact, contrary to traditional angularly resolved photoelectron spectroscopy [3], in wave function microscopy one is interested in the radial rather than the angular electron distribution. In the early 1980s an experiment was proposed [4] where electron waves interacting with a static electric field could be imaged as long as the de Broglie wavelength of these electrons is large enough. This was realized by the so-called photodetachment microscope [5] that was experimentally implemented soon after the emergence of photoelectron imaging techniques [6-8].

The above proposal was further developed $[9,10]$ so as to include atomic photoionization and the possibility of visualizing electron standing waves corresponding to quasidiscrete electronic states of an atom. Photoionization is very different from photodetachment since photoionized electrons interact with both the electric field and the Coulomb field of the residual ion and neither field can be considered as a perturbation. The first photoionization microscopy experiments were performed with Xe atoms [11-13]. Remarkably, in these experiments [14] the number and position of nodes of the recorded wave function evolved smoothly with energy, and remained to a large extent insensitive to the substantial number of resonances lying in the examined energy range [15]. Thus, only the electron wave function associated with the ionization continuum was observed, while the original idea of obtaining an image related to a quasibound quantum state $[9,10]$ was not realized. Here, we present the first realization of the experiment proposed in Refs. $[9,10]$ by photoionizing $\mathrm{Li}$ atoms in the presence of a $\sim 1 \mathrm{kV} / \mathrm{cm}$ static electric field. Contrary to the earlier observations in $\mathrm{Xe}$, the $\mathrm{Li}$ wave function microscopy images are sensitive to the presence of resonances and therefore directly reveal the nature of the quasibound states.

Before presenting our findings it is instructive to qualitatively discuss the concept of wave function microscopy, which can be understood on the basis of hydrogenic Stark theory. For a hydrogen atom in a static field of strength $F$, directed along the $z$ axis, the Hamiltonian is separable in parabolic coordinates $\xi=r+z, \eta=r-z$ and $\varphi=$ $\arctan (y / x)$. Separability along $\varphi$ leads to the azimuthal quantum number $m$, while separability along $\xi$ and $\eta$ involves two separation constants, $\beta_{1}$ and $\beta_{2}=1-\beta_{1}$, 
and leads to parabolic quantum numbers $n_{1}$ and $n_{2}$, counting the nodes in the $\xi$ - and $\eta$-part of the wave function, respectively [16-18]. The electron is always bound along the $\xi$ coordinate (see Fig. 1). The energy range of interest here lies between the field-free ionization limit $(E=0)$ and the classically calculated saddle point energy, $E_{\mathrm{sp}}=$ $-2 \sqrt{F}$ a.u. (atomic units: $\hbar=m_{e}=e=1$ ), that is the energy to which the ionization limit is lowered due to the presence of the field. Within this range the electron can escape in the negative $z$ direction along the $\eta$ coordinate. For $E_{\mathrm{sp}} \leq E \leq 0$ each $n_{1}$ is associated with a threshold $E_{\mathrm{thr}}^{n_{1}}$ (found by solving the equation $E=-2\left[\beta_{2}\left(E, n_{1}\right) F\right]^{1 / 2}$ ) and $n_{2}$ is meaningful only when $E<E_{\mathrm{thr}}^{n_{1}}$. Each $\left(n_{1}, n_{2}\right)$ pair corresponds to a quasibound state where the electron escapes solely via tunneling through the $\eta$ potential [Fig. 1(a)]. For $E>E_{\text {thr }}^{n_{1}}$, where $n_{2}$ loses its meaning, the electron escapes over the $\eta$-potential barrier [Fig. 1(b)]. Thus, within $E_{\mathrm{sp}} \leq E \leq 0$ quasidiscrete and continuum Stark states with different $n_{1}$ values coexist. Wave function microscopy aims at recording the photocurrent density $j(\xi)$ at a macroscopic distance (see Fig. 1) [10]. Let us first consider the absence of resonances and denote by $n_{1}^{0}$ the largest $\xi$-quantum number corresponding to a continuum (for a given energy). This, so-called, background (BG) density, may be written as
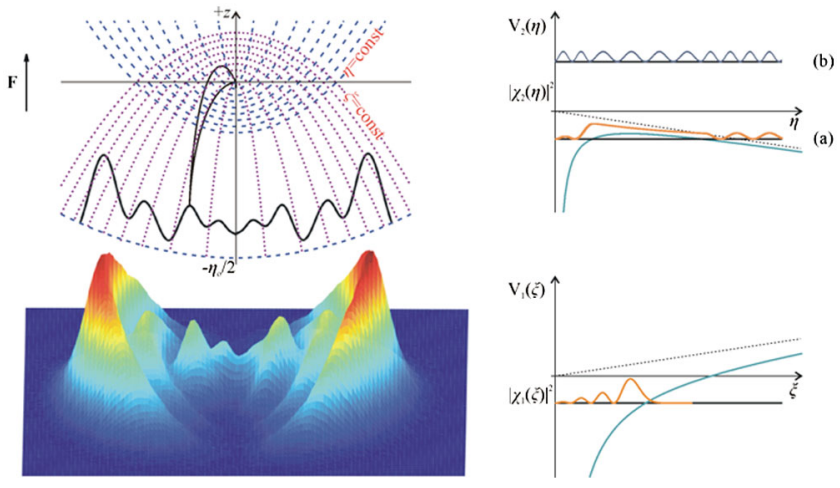

FIG. 1 (color online). Wave function microscopy aims at observing the spatial structure of the electronic wave function. The electron flux stemming from the photoionization of an atom in the presence of a static electric field is recorded perpendicularly to the field and at macroscopic distance, i.e., approximately along a given constant $\eta=\eta_{0}$ paraboloid. The image corresponds to the wave function along the $\xi$ coordinate where the electron motion is always bounded. The nodes of the wave function are clearly observed in the example shown, recorded with $F=1010 \mathrm{~V} / \mathrm{cm}$ and electron excitation energy exceeding $E_{\text {sp }}$ by $10 \mathrm{meV}$. Depending on the electron energy with respect to the maximum of the $\eta$ potential, the classical electron motion may be either bounded (a) or free (b). In the former case the electron can escape solely via tunneling and the image corresponds to a direct macroscopic projection of a quantum standing wave characterizing the quasibound electronic state $\left(n_{1}^{\text {res }}, n_{2}^{\text {res }}\right.$, $m$ ) where the electron is initially localized within the inner $\eta$-potential well.

$$
j_{\mathrm{BG}}\left(\xi, \eta=\eta_{0}\right) \propto\left|\sum_{n_{1}=0}^{n_{1}^{0}} c_{n_{1}} \chi_{1, n_{1}}(\xi)\right|^{2},
$$

where $\chi_{1, n_{1}}$ denotes wave functions along the $\xi$ coordinate and $c_{n_{1}}$ the corresponding weights. It turns out that $j_{\mathrm{BG}}\left(\xi, \eta=\eta_{0}\right)$, having the form of an interferogram, is dominated by $\chi_{1, n_{1}^{0}}$. Therefore, the density of Eq. (1) exhibits $n_{1}^{0}$ dark fringes, which increase smoothly with energy. The presence of a single quasidiscrete state with a wave function $\chi_{1, n_{1}^{\text {res }}}(\xi)\left(n_{1}^{\text {res }}>n_{1}^{0}\right)$ modifies $j(\xi)$ according to

$$
\begin{aligned}
& j_{\mathrm{BG}+\mathrm{RES}}\left(\xi, \eta=\eta_{0}\right) \\
& \quad \propto\left|\sum_{n_{1}=0}^{n_{1}^{0}} c_{n_{1}} \chi_{1, n_{1}}(\xi)+a_{n_{1}^{\mathrm{res}}, n_{2}} \chi_{1, n_{1}^{\mathrm{res}}}(\xi)\right|^{2},
\end{aligned}
$$

where the weight $a_{n_{1}^{\text {res }}, n_{2}}$ depends on $n_{2}$. The latter, however, cannot, in principle, be extracted from $j_{\mathrm{BG}+\mathrm{RES}}\left(\xi, \eta=\eta_{0}\right)$. For a sufficiently narrow resonance, $\chi_{1, n_{1}^{\text {res }}}$ dominates the interferogram. Therefore, $j_{\mathrm{BG}+\mathrm{RES}}\left(\xi, \eta=\eta_{0}\right)$ is expected to evolve nonmonotonically in the vicinity of resonances and hydrogenic Stark theory [10] predicts (i) A strong onresonance modification of the interference pattern that can include a fringe number change. Hence, $j_{\mathrm{BG}+\mathrm{RES}}\left(\xi, \eta=\eta_{0}\right)$ can exhibit $n_{1}^{\text {res }}$ dark fringes while their number is reduced to $n_{1}^{0}$ at energies below and just above the resonance. (ii) A modulation of the fringe contrast due to the coherent superposition of resonant and nonresonant contributions in Eq. (2). (iii) A broadening of the image outer ring, associated to tunneling ionization [19].

The ultimate goal of photoionization microscopy is to uncover the features of $j\left(\xi, \eta=\eta_{0}\right)$ stemming from the resonant state $\chi_{1, n_{1}^{\text {res }}}$ that is to say, to directly observe the wave function projection corresponding to a quasibound electronic state $\left(n_{1}^{\text {res }}, n_{2}, m\right)$. In nonhydrogenic atoms, however, the above features are obscured due to shortrange interactions occurring when the excited electron penetrates the ionic core. This leads to mixing between states of different $n_{1}$. Therefore, even if the system is

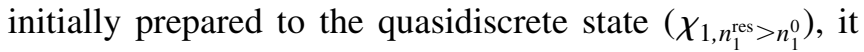
may "leak" to the degenerate continua $\left(\chi_{1, n_{1} \leq n_{1}^{0}}\right)$ and the resonant character may not show-up in $j\left(\xi, \eta=\eta_{0}\right)$. This was the case in the xenon experiment. Obviously, the effect would be expected in experiments on hydrogen atoms. Nevertheless, the observation of resonant effects in nonhydrogenic atoms appears to be of fundamental significance, as it turns photoionization microscopy into a more general technique, eventually capable of dealing with polyatomic systems. In order to overcome the problem linked to the large core penetration encountered in high- $Z$ atoms, we use the low- $Z$ alkali Li atom, which allows, for the first time, undisputable experimental signatures of resonant effects. The degree of core penetration can be assessed by a comparison of the magnitude of the quantum defects 
$\mu_{\ell}$ for the $n s, n p$, and $n d$ Rydberg series: $\mathrm{Xe}\left(\mu_{s} \approx 4.0\right.$, $\mu_{p} \approx 3.5$ and $\left.\mu_{d} \approx 2.4\right)$ and $\operatorname{Li}\left(\mu_{s} \approx 0.4, \mu_{p} \approx 0.05\right.$ and $\left.\mu_{d} \approx 0.002\right)$ [20] is revealing. In order to increase the selectivity, we use single-photon excitation and ionization of $\mathrm{Li}$ and linear perpendicular laser polarization that leads to $|m|=1$ final states which are expected to be closer to the hydrogenic case because the final Stark state wave function does not contain any $\ell=0$ core-penetrating component.

Our microscope is similar to the one employed earlier $[11,13,14,21]$. It is based on a standard velocity-map imaging design [22]. Image magnification is accomplished via an einzel lens $[23,24]$. The field accelerates photoelectrons towards a position-sensitive detector (MCP + phosphor-screen + CCD camera). The Li atomic jet is produced by laser ablation of a pure Li metallic rod. The $\mathrm{Li}$ atoms are carried by a pulsed $\mathrm{He}$ gas jet. The jet is skimmed and interacts with tunable UV laser radiation delivered by a high finesse OPO system (Spectra-Physics MOPO-pulse energy $\sim 1 \mathrm{~mJ}$, pulse duration $\sim 5 \mathrm{~ns}, 10 \mathrm{~Hz}$ repetition rate, bandwidth $0.05 \mathrm{~cm}^{-1}$ ).

Experimentally, the electronic wave function of a resonance should be easier to visualize when the resonances dominate the ionization yield and the number of nodes in the wave function $\left(n_{1}{ }^{0}\right.$ and $\left.n_{1}{ }^{\text {res }}\right)$ is small. These two criteria are met just above $E_{\mathrm{sp}}$. Interferograms recorded with our microscope for ionization of $\mathrm{Li}$ just above the saddle point $\left(F=1010 \mathrm{~V} / \mathrm{cm}=1.96 \times 10^{-7}\right.$ a.u., $\varepsilon \equiv$ $\left.E /\left|E_{\mathrm{sp}}\right|=-0.928\right)$ are shown in the top half of Fig. 2. Their angular distributions reflect the excitation of the $p$ component of the Stark states, as expected for a singlephoton transition from an $s$ state. Figure 2(c)-(2) corresponds to the measurement at a $\left(n_{1}{ }^{\text {res }}=2, n_{2}=25, m=1\right)$ resonance [18], and Figs. 2(c)-(1) and 2(c)-(3) correspond to recordings $120 \mu \mathrm{eV}$ below $(\varepsilon=-0.933)$ and above $(-0.923)$ this resonance. In nonresonant ionization, the number of dark fringes should remain unchanged and equal to $n_{1}{ }^{0}=1$, for this energy range. On resonance, however, we do observe two dark fringes [Fig. 2(c)-(2)]. Hence, we measure a direct projection of the squared $\xi$-wave function component of the $\left(n_{1}{ }^{\text {res }}=2, n_{2}=25, m=1\right)$ state (located $2 \times 10^{-7}$ a.u. below the $\eta$-potential barrier [Fig. 1(a)]).

In Figs. 2(c), experimental images are compared to calculations based on wave packet propagation techniques, solving the time-dependent Schrodinger equation [25]. The quantum defects are taken into account using a model potential [26]. One clearly observes that theory also predicts two dark fringes on resonance, and a single one below and above it. In Fig. 2(d), the comparison between $\mathrm{Li}$, hydrogen $(\mathrm{H})$, and cesium (Cs) shows a single dark fringe below [Fig. 2(d)-(1)] and above the resonance [Fig. 2(d)-(3)]. However, on the resonance, the nearly identical interferograms for $\mathrm{Li}$ and $\mathrm{H}$ show two dark fringes, whereas the Cs interferogram exhibits just a single one [Fig. 2(d)-(2)].
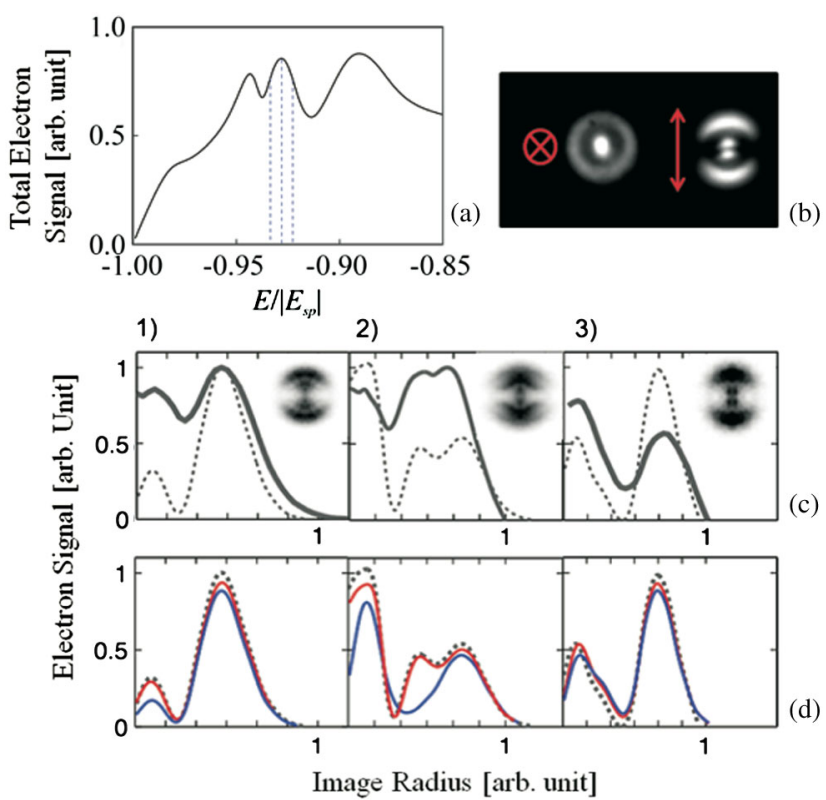

FIG. 2 (color online). (a) Measured ionization yield versus energy with respect to the saddle point showing Stark resonances $\left(F=1010 \mathrm{~V} / \mathrm{cm}=1.96 \times 10^{-7}\right.$ a.u., $E_{\mathrm{sp}}=-8.87 \times 10^{-4}$ a.u. $=$ $-24.11 \mathrm{meV}),|m|=1$ ). Images, shown in (c) below, are measured across one resonance near threshold (indicated by dashed blue lines). (b) Measured images. For $m=0$ (laser polarization parallel to the electric field) the angular distribution is isotropic. For $|m|=1$ the distribution is aligned along the laser polarization. (c) Measured (continuous line) and corresponding calculated (dotted line) radial distributions obtained from one-photon ionization of $\mathrm{Li}(|m|=1)$ using $F=1010 \mathrm{~V} / \mathrm{cm}$ (measured images are presented as insets) at three different photon energies, namely on resonance case (2) $1.74 \mathrm{meV}$ above the saddle point $\left(\varepsilon \equiv E /\left|E_{\mathrm{sp}}\right|=-0.928, \quad \lambda=230.912 \mathrm{~nm}\right.$ and off-resonance cases (1) and (3) $120 \mu \mathrm{eV}$ below and above the resonance, $\lambda=230.917 \mathrm{~nm} \lambda=230.906 \mathrm{~nm}$, respectively. An additional on resonance fringe corresponding to an increase of the nodes of the wave function is clearly seen. The interferogram is a direct image of the $\left(n_{1}^{\text {res }}=2,|m|=1\right)$ state and it is similar to the calculated one. (d) Calculated radial distributions for $\mathrm{H}$ (red line), Li (black-dotted line), and Cs (blue line) $120 \mu \mathrm{eV}$ below (1), on (2), and $120 \mu \mathrm{eV}$ above (3) the $\left(n_{1}^{\text {res }}=2, m=1\right)$ resonance.

This is due to the fact that, for $|m|=1, \mathrm{Li}$ is almost perfectly hydrogenic and thus its quasibound and continuum states are nearly orthogonal. In Cs, by contrast, interchannel coupling leads to population "leaking" from the quasibound state to the degenerate continuum. This conclusion, however, should not be generalized since theoretical predictions showed that, even for Xe, resonant effects could be observable, albeit at a much higher spectral and spatial resolution [27]. We further note that wave packet calculations reproduce qualitatively the experimental relative fringe intensities in $\mathrm{Li}$. A number of reasons could be responsible for the lack of quantitative agreement (e.g., spatial resolution, polarization imperfections, or field inhomogeneity). Computationally, it is difficult to include all 
(a)
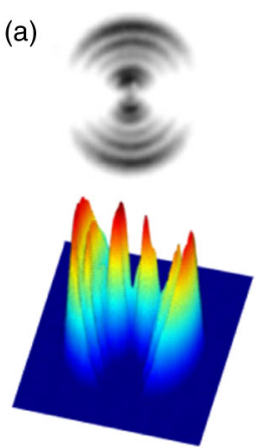

I)
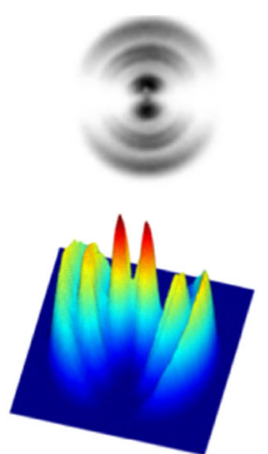

2)

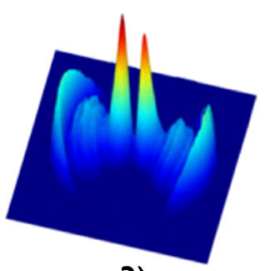

3)

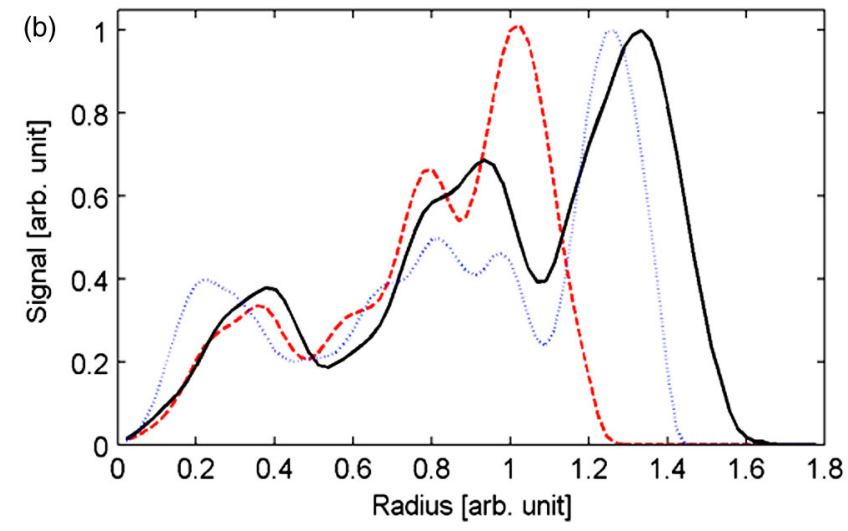

FIG. 3 (color online). (a) 2D and 3D representation of measured photoelectron images obtained in one-photon ionization of $\mathrm{Li}$ with $F=1010 \mathrm{~V} / \mathrm{cm}$, around the $\left(n_{1}^{\text {res }}=6, m=1\right)$ resonance $\left(\varepsilon \equiv E /\left|E_{\mathrm{sp}}\right|=-0.789\right)$. (1) $-19.78 \mathrm{meV} \quad(\lambda=$ $230.800 \mathrm{~nm}) ; \quad$ (2) $\quad-19.07 \mathrm{meV} \quad(\lambda=230.769 \mathrm{~nm}) ; \quad$ and (3) $-18.49 \mathrm{meV}(\lambda=230.744 \mathrm{~nm})$. (b) Radial distribution of the images. The radius is scaled to the classical radius $\mathrm{R}_{\max }^{c l}$ [12], whose size on the detector phosphor screen is $\sim 6 \mathrm{~mm}$. While in the non-resonant case, the radius of the image should smoothly increase with photon energy, we observe that the image is larger on-resonance (black continuous curve) and smaller at both lower (red-dashed line) and higher (blue-dotted line) photon energies. This is attributed to the tunneling ionization through the barrier.

these effects. In addition, the model takes only implicitly into account the excitation process. Therefore, a more accurate description of the threshold ionization mechanism is compulsory. A recently developed theoretical treatment, successfully reproducing our nonresonant data of $\mathrm{Li}$ [28], looks promising towards this direction.

Further experiments at the same field but near the $\left(n_{1}{ }^{\text {res }}=6, n_{2}, m=1\right)$ resonance, located at $\varepsilon=-0.789$ $\left(\sim E_{\mathrm{sp}}+5 \mathrm{meV}\right)$, are shown in Fig. 3(a). The main effect of the resonance is a dramatic change in the radial interference pattern, which is relatively smooth on both its red and blue side ( $620 \mu \mathrm{eV}$ below and above it), while, on resonance, it is dominated by two rings that appear at intermediate radii between the broad outer ring and the central peak. This is a consequence of the aforementioned coherent superposition of resonant and non-resonant contributions, expressed by Eq. (2). We notice that the width of the outer ring of the image is much broader onresonance. This is more clearly visible in Fig. 3(b), showing the cross section of the radial distribution of emitted electrons as a function of the scaled radius, defined as the ratio of experimental radius divided by the classical maximum radius of impact. The effect was recently predicted by Zhao et al. [19]. It corresponds to an on resonance increase of the probability for the electron to tunnel through the potential barrier as a result of its high localization near the edge of the potential. This holds for either hydrogenic or nonhydrogenic Rydberg atoms (the barrier is present in both cases).

In conclusion, we have presented the first experimental wave function microscopy images where a projection of a quasidiscrete electronic state $\left(n_{1}, n_{2}, m\right)$ is directly visualized. The evolution of the interference pattern shows an on-resonance change of the number of fringes and, consequently, of the parabolic quantum number $n_{1}$. This is in agreement with the expectations of the experiment proposed during the 1980s $[9,10]$. Further, the observed on resonance broadening of the outer image ring confirms recent theoretical predictions [19] associated with tunneling through the barrier. Finally, wave function microscopy appears as a powerful tool for investigating matter on the quantum level that brings information on the electron's presence within the inner $\eta$-potential well. We hope that the present work will motivate further experimental and theoretical developments.

*franck.lepine@univ-lyon1.fr

[1] J. Itatani, J. Levesque, D. Zeidler, H. Niikura, H. Pépin, J. C. Kieffer, P. B. Corkum, and D. M. Villeneuve, Nature (London) 432, 867 (2004).

[2] P. Puschnig, S. Berkedile, A. J. Flemming, G. Koller, K. Emtsev, T. Seyller, J. D. Riley, C. Ambrosch-Draxl, F. P. Netzer and M. Ramsey, Science 326, 702 (2009).

[3] K. Reid, Annu. Rev. Phys. Chem., 54, 397 (2003).

[4] I. I. Fabrikant, JETP 52, 1045 (1980).

[5] C. Blondel, C. Delsart, and F. Dulieu, Phys. Rev. Lett. 77, 3755 (1996).

[6] A. J. R. Heck and D. W. Chandler, Annu. Rev. Phys. Chem. 46, 335 (1995); C. Bordas, F. Paulig, H. Helm, and D. L. Huestis, Rev. Sci. Instrum. 67, 2257 (1996).

[7] C. Blondel, W. Chaibi, C. Delsart, C. Drag, F. Goldfarb, and S. Kröger, Eur. Phys. J. D 33, 335 (2005).

[8] F. Goldfarb, C. Drag, W. Chaibi, S. Kröger, C. Blondel, and C. Delsart, J. Chem. Phys. 122, 014308 (2005).

[9] Yu. N. Demkov, V. D. Kondratovich, and V. N. Ostrovsky, Pis'ma Zh. Eksp. Teor. Fiz. 34, 425 (1981) [JETP Lett. 34, 425 (1981)].

[10] V. D. Kondratovich and V. N. Ostrovsky, J. Phys. B 17, 1981 (1984); 17, 2011 (1984); 23, 21 (1990); 23, 3785 (1990).

[11] C. Nicole, I. Sluimer, F. Rosca-Pruna, M. Warntjes, M. J. J. Vrakking, Ch. Bordas, F. Texier, and F. Robicheaux, Phys. Rev. Lett. 85, 4024 (2000). 
[12] C. Bordas, Phys. Rev. A 58, 400 (1998).

[13] C. Nicole, H. L. Offerhaus, M. J. J. Vrakking, F. Lépine, and Ch. Bordas, Phys. Rev. Lett. 88, 133001 (2002).

[14] F. Lépine, C. Bordas, C. Nicole, and M. J. J. Vrakking, Phys. Rev. A 70, 033417 (2004).

[15] C. Bordas, F. Lépine, C. Nicole, and M. J. J. Vrakking, Phys. Rev. A 68, 012709 (2003).

[16] H. A. Bethe and E. E. Salpeter, Quantum Mechanics of One and Two-Electron Atoms (Springer, Berlin, 1957).

[17] E. Luc-Koenig and A. Bachelier, J. Phys. B 13, 1743 (1980); 13, 1769 (1980).

[18] D. A. Harmin, Phys. Rev. A 24, 2491 (1981); 26, 2656 (1982).

[19] L.B. Zhao and J.B. Delos, Phys. Rev. A 81, 053417 (2010); 81, 053418 (2010).

[20] Y. Ralchenko, A. Kramida, and J. Reader (NIST ASD Team), NIST Atomic Spectra Database (version4.1) (2011), http://physics.nist.gov/asd.
[21] F. Lépine, S. Zamith, A. de Snaijer, C. Bordas, and M. J. J. Vrakking, Phys. Rev. Lett. 93, 233003 (2004).

[22] A. T. J. B. Eppink and D. H. Parker, Rev. Sci. Instrum. 68, 3477 (1997).

[23] H. L. Offerhaus, C. Nicole, F. Lépine, C. Bordas, F. RoscaPruna, and M. J. J. Vrakking, Rev. Sci. Instrum. 72, 3245 (2001).

[24] M. M. Harb, S. Cohen, E. Papalazarou, F. Lépine, and C. Bordas, Rev. Sci. Instrum. 81, 125111 (2010).

[25] F. Robicheaux and J. Shaw, Phys. Rev. Lett. 77, 4154 (1996).

[26] M. Aymar, S. Feneuille, and M. Klapisch, Nucl. Instrum. Methods 90, 137 (1970).

[27] F. Texier, Phys. Rev. A 71, 013403 (2005).

[28] L. B. Zhao, I. I. Fabrikant, J. B. Delos, F. Lépine, S. Cohen, and C. Bordas, Phys. Rev. A 85, 053421 (2012). 